\title{
Management of Tricuspid Regurgitation: The Role of Transcatheter Therapies
}

\author{
Maurizio Taramasso, Christelle Calen, Andrea Guidotti, Shingo Kuwata, Hector Rodriguez Cetina Biefer, \\ Fabian Nietlispach, Michel Zuber and Francesco Maisano
}

${ }^{1}$ Heart Valve Clinic, University Hospital of Zurich, University of Zurich, Zurich, switzerland

\begin{abstract}
Surgical treatment is the gold standard treatment of functional tricuspid regurgitation (FTR) but this carries high risks of morbidity and mortality. Percutaneous procedures are an attractive alternative to surgery for selected patients deemed to be high-risk surgical candidates. A number of tricuspid transcatheter devices have been developed to treat FTR, but at present, evidence of their efficacy and safety is scarce. Preliminary data have shown promising results, but ongoing and future studies will provide a clearer picture of the benefits of these new techniques.
\end{abstract}

\section{Keywords}

Tricuspid regurgitation, transcatheter, annuloplasty, tricuspid valve spacer

Disclosure: The authors have no conflicts of interest to declare.

Received: 4 October 2016 Accepted: 9 March 2017 Citation: Interventional Cardiology Review 2017;12(1):51-5. DOI: 10.15420/icr.2017:3:2

Correspondence: Maurizio Taramasso, Cardiovascular Surgery Department, University Hospital of Zurich, Rämistrasse 100, CH-8091 Zürich, Switzerland.

E: Maurizio.Taramasso@usz.ch.

The leading aetiology of tricuspid regurgitation (TR) in developed countries is functional, secondary to left side heart disease and pulmonary hypertension. ${ }^{1}$ Currently, moderate-to-severe TR affects approximately 1.6 million patients in the United States, of whom only 8,000 undergo tricuspid surgery annually: ${ }_{i}^{2}$ this results in an extremely large number of untreated patients with significant TR, a condition that has been clearly associated with risk of mortality and cardiovascular events. ${ }^{3}$

The high prevalence of TR is in part due to the undertreatment as TR was wrongly considered a benign and reversible condition in the past. In fact, due to the incorrect concept that TR would disappear once the primary left heart disease (LHD) had been treated, surgical avoidance of tricuspid valve (TV) repair was for many years easily accepted in patients with functional tricuspid regurgitation (FTR). Thereafter, many investigators progressively reported findings in favour of a more aggressive surgical approach to FTR. Furthermore, the most recent guidelines recommend surgical repair of concomitant TR during left valve surgery in patients with tricuspid annular dilatation with non-severe TR, to reduce the occurrence of late TR in patients who underwent LHD surgery. ${ }^{4,5}$

TV surgery is associated with a high risk of morbidity and mortality in cases of TR recurrence after left heart surgery (up to $25 \%$ in some series), particularly because these patients are typically referred to surgical treatment only when they develop severe incapacitating symptoms of right heart failure and organ dysfunction. ${ }^{6,7}$

Percutaneous procedures are an attractive alternative to surgery for patients deemed to be high-risk surgical candidates. Numerous tricuspid transcatheter devices have been developed to treat FTR. At present, clinical experiences with transcatheter TV therapies are still preliminary, and data to support their efficacy are scarce. However, initial experiences are promising and the safety profiles of the different devices favourably compares to that of conventional surgical treatment. ${ }^{7}$

This review will explore the available technologies that are currently under evaluation and their preliminary clinical results.

\section{Anatomy and Pathophysiology of Functional Tricuspid Regurgitation: Interventional Considerations}

A deep knowledge of the complex anatomy of the TV is a mandatory pre-requisite to understand the challenges encountered in developing percutaneous tricuspid therapy.

The normal TV apparatus is composed of three leaflets (septal, posterior and anterior), chordae tendineae, and two or three welldeveloped papillary muscles (see Figure 1). Several structures of major surgical importance surround the TV, such as the right coronary artery, the atrioventricular (AV) node, the bundle of His and the coronary sinus.

The non-planar and non-circular structure of the tricuspid annulus must be considered when considering tricuspid repair technologies. The normal shape of the tricuspid annulus is semi-lunar and it is larger compared with the mitral one. In physiological conditions, the tricuspid annulus has a non-planar three-dimensional saddle-shape configuration. Such a characteristic is absent in cases of functional TR, in which the annulus becomes enlarged, more circular and flattened. Annular dilatation occurs mainly in its anterior-posterior aspect. The consequence of severe functional TR is further right ventricular (RV) dilatation with progressive papillary muscles displacement and consequent tethering of the leaflets.

The development of FTR can be classified into three progressive stages of the disease (see Figure 2):8,9 
Figure 1: Anatomy of the Tricuspid Valve in a Cadaveric Heart: Surgical View

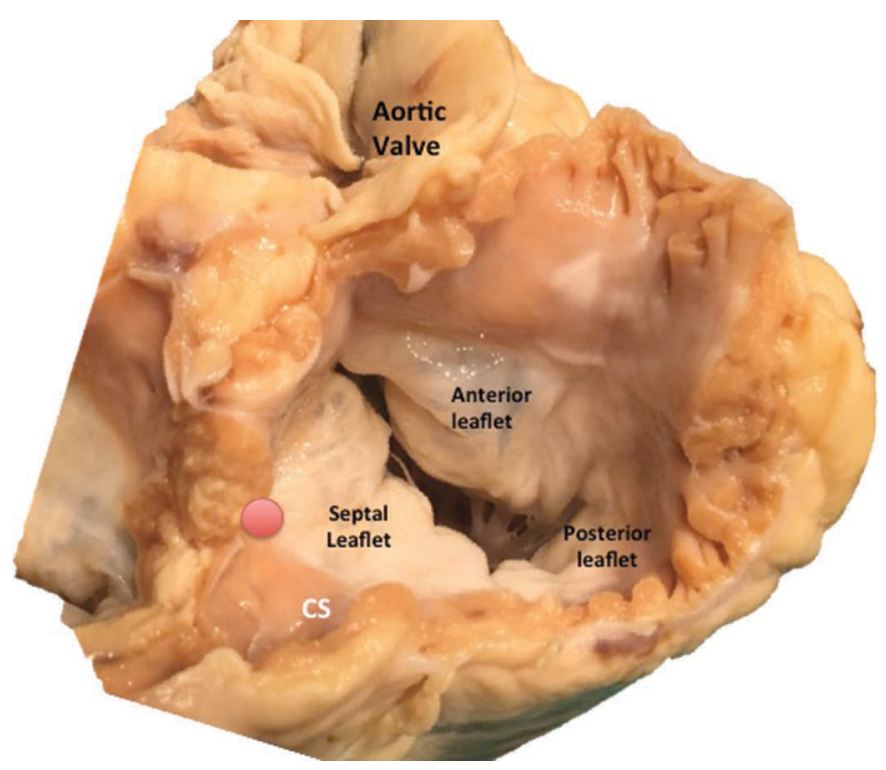

The red spot indicates the location of the atrioventricular node; $C S$ = coronary sinus.

Figure 2: Pathophysiology of Functional Tricuspid Regurgitation

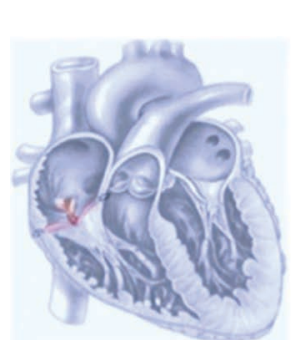

Phase I

Initial Right Ventricular (RV)

dilatation results in Tricuspid Annulus (TA) dilatation

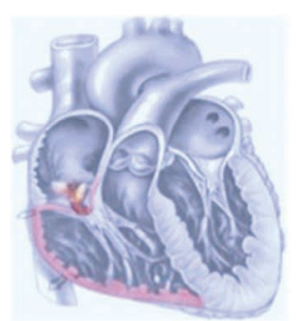

Phase II Progressive RV and TA dilatation results in lack of leaflets coaptation

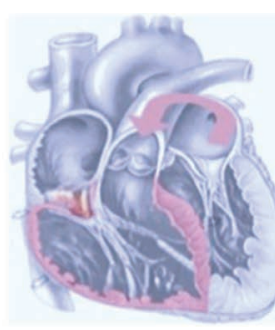

Phase III Progressive RV distortion results in tethering of the hypertension
hy
Figure modified from Taramasso et al. Eur Heart J. 2016 Jan 21. pii: ehv766.

- Stage 1: annular dilatation. FTR is invariably associated with tricuspid annular dilatation, typically secondary to RV enlargement. Annular dilatation can be also initiated by right atrial enlargement, especially in patients with chronic atrial fibrillation. In the first phase of the disease, the tricuspid annulus is dilated, but the degree of TR is not yet severe.

- Stage 2: progressive TV annulus enlargement prevents normal leaflet coaptation. Dilatation of the tricuspid annulus is not symmetrical; it occurs mainly in its anterior and posterior segments, which corresponds to the free wall of the RV. In this phase, FTR severity become significant and there is initial RV dilatation and deformation.

- Stage 3: RV dilatation and dysfunction, and TV tethering. The consequence of severe FTR is progressive RV dilatation and dysfunction. Progressive RV dilatation causes displacement of papillary muscles and consequent leaflet tethering.

\section{The Role of Interventional Tricuspid Regurgitation Therapy: Treatment and 'Prevention'}

Patients who could benefit from transcatheter treatment of TR are those with symptomatic TR irrespective of maximal medical therapy or with initial signs of RV dysfunction, who are deemed to be either at high risk for conventional open heart surgery or inoperable.

Some of the concepts that have been developed for the percutaneous treatment of mitral regurgitation may be adapted to percutaneous repair of the TV.

Since the initial mechanism leading to FTR is annular dilatation was elucidated, most of the current transcatheter technologies aim to fix annular dilatation by means of a tricuspid annuloplasty, the gold standard surgical treatment in this setting.

Once the safety profile of percutaneous tricuspid annuloplasty becomes proven, a concomitant tricuspid valve repair during transcatheter mitral valve intervention will likely be justified even in Stage 1 of the disease (significant annular dilatation even in absence of severe TR), to reduce the risk of late TR occurrence, similarly to what is recommended for cases of concomitant surgical treatment of TR during left valve surgery. This is merely a speculative assumption in the absence of supportive data, but such an aggressive treatment of concomitant TR could, in the near future, mimic the present surgical management approach.

\section{Transcatheter Repair Techniques}

\section{Annuloplasty Devices}

The TriCinch ${ }^{\mathrm{TM}}$ system

The TriCinch ${ }^{\mathrm{TM}}$ system (4Tech Cardio Ltd) is a percutaneous device designed to cinch and remodel the tricuspid annulus through a transfemoral venous access. ${ }^{10,11}$ Annular dimension reduction and consequent improvement of leaflet coaptation is achieved by means of stainless steel corkscrew fixation into the anteroposterior TV annulus (see Figure 3A). The ideal site for anchor implantation is defined by a careful analysis of the computed tomography (CT) scan, usually close to the anteroposterior commissure. The corkscrew is connected through a dacron band to a self-expanding nitinol stent. Following corkscrew implantation, the device is pulled caudally to bring the anterior annulus towards the septum, until the desired reduction of TR is observed during live echocardiography. Optimal tension is maintained by the delivery of a nitinol self-expanding stent in the inferior vena cava. The size of the stent is evaluated on a pre-procedural CT scan. The procedure is carried out under general anaesthesia, with transoesophageal echocardiography (TEE) and/or intracardiac echocardiography (ICE) guidance. A coronary wire is positioned in the right coronary artery during the procedure and its patency is confirmed with ad hoc injections. First-in-man data for this procedure were reported in 2016.10 The multicentre Percutaneous Treatment of Tricuspid Valve Regurgitation With the Tricinch System (PREVENT) feasibility and safety trial (NCT02098200) is ongoing in Europe. CE mark approval is expected in 2017.

\section{The Trialign ${ }^{\mathrm{TM}}$ system}

The Trialign ${ }^{\mathrm{TM}}$ system (Mitralign Inc.) is designed to perform a percutaneous suture annuloplasty of the TV, replicating the surgical bicuspidation technique, which is based on reducing annular dilatation and improving TV competency by plication of the posterior tricuspid annulus (see Figure 3B). ${ }^{12,13}$ With the Trialign device, the posterior leaflet is plicated by means of a pair of pledgeted sutures, which are implanted transanular from the ventricle to the atrium. Through right jugular access, the system is advanced in the right atrium, and then across the TV into the RV on fluoroscopic and TEE guidance. 

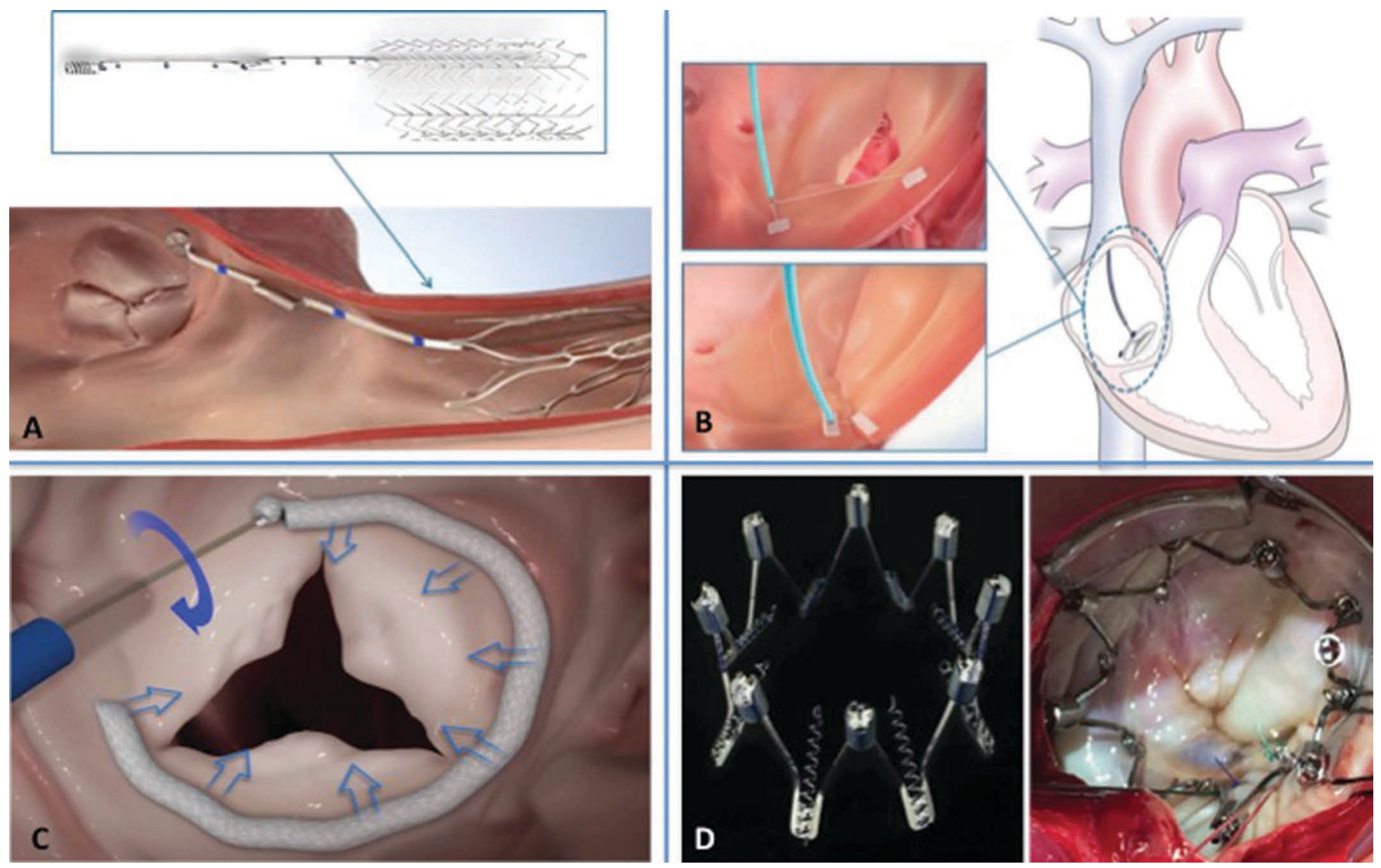

Part of this figure is modified from Taramasso et al. Eur Heart J. 2016 Jan 21. pii: ehv766.

The catheter is articulated under the annulus towards the anteroposterior commissure. The catheter tip is positioned approximately $2-5 \mathrm{~mm}$ from the hinge point of the leaflet and a wire is advanced with radiofrequency across the annulus into the right atrium (RA), where it is snared and externalised. This wire is then used to advance the pledget-delivery catheter across the annulus into the RV. The pledget is then delivered and compressed so that it is positioned on both the ventricular and atrial sides of the annulus. These steps are repeated on the opposite commissure of the posterior leaflet. The two pledgets on the anteroposterior and posteroseptal commissure are then pulled together and locked in place to plicate the posterior annulus and bicuspidalise the TV.

The Trialign system is currently undergoing evaluation in prospective multicentre US feasibility of a Percutaneous Tricuspid Valve Annuloplasty System for Symptomatic Chronic Functional Tricuspid Regurgitation (SCOUT) (NCT02574650) and European CE-mark (SCOUT-II) studies. The distinctive features of the Trialign system are that it is based on a surgical technique, the footprint of the final implant is small, the leaflets remain untouched, and future access to the TV for pacing or transcatheter treatment is maintained.

\section{Cardioband Tricuspid}

The Cardioband system (Valtech Cardio) is a transfemoral fully percutaneous direct annuloplasty device, which gained CE mark approval in 2015 for the treatment of high-risk patient with functional mitral regurgitation. ${ }^{14,15}$ In the standard procedure, a surgical-like band is delivered through trans-septal access in the left atrium and implanted on the atrial side of the posterior mitral annulus, from lateral to medial trigon, by means of multiple anchor elements. Final adjustment of the
Cardioband size under echocardiography and fluoroscopic guidance provides a reduction of the septolateral diameter of the mitral valve, with consequent improvement of leaflet coaptation and reduction of valve regurgitation. A feasibility trial has demonstrated safety and efficacy of the Cardioband in the mitral position. ${ }^{14}$ The same concept has been recently used to treat FTR, as the Cardioband device closely reproduces the gold standard surgical treatment, ring annuloplasty. The same device is used by means of a transfemoral venous approach. A slightly modified delivery system is used, with different steering curves compared with the mitral procedure, to facilitate the implant on the tricuspid annulus. The band is implanted on the mural part of the tricuspid annulus, from the anteroseptal commissure to the initial part of the septal annulus. Final adjustment provides a marked reduction in the anteroseptal valve diameter and reduction of TR by forcing the leaflet coaptation (see Figure $3 \mathrm{C}$ ). Clinical experience with the Cardioband system in the tricuspid position is limited; to date, only five patients have been treated (unpublished data).

\section{The Millipede system}

The Millipede annular ring (Millipede) is a direct annuloplasty device that has been developed to treat TR and mitral regurgitation. This device mimics surgical annuloplasty by transcatheter implantation of an expandable and contractable ring that uses a novel attachment technique with many small, barbed anchors to secure it in place on the atrial side of the tricuspid annulus (see Figure 3D). Prior to ring fixation, the annulus is expanded by a dilator to the size and shape of the ring. The ring is then anchored to the annulus and contracts to a predefined size, therefore resulting in size reduction of the annulus. Early first-in-human Phase I studies of this procedure took place in 


\section{Structural}

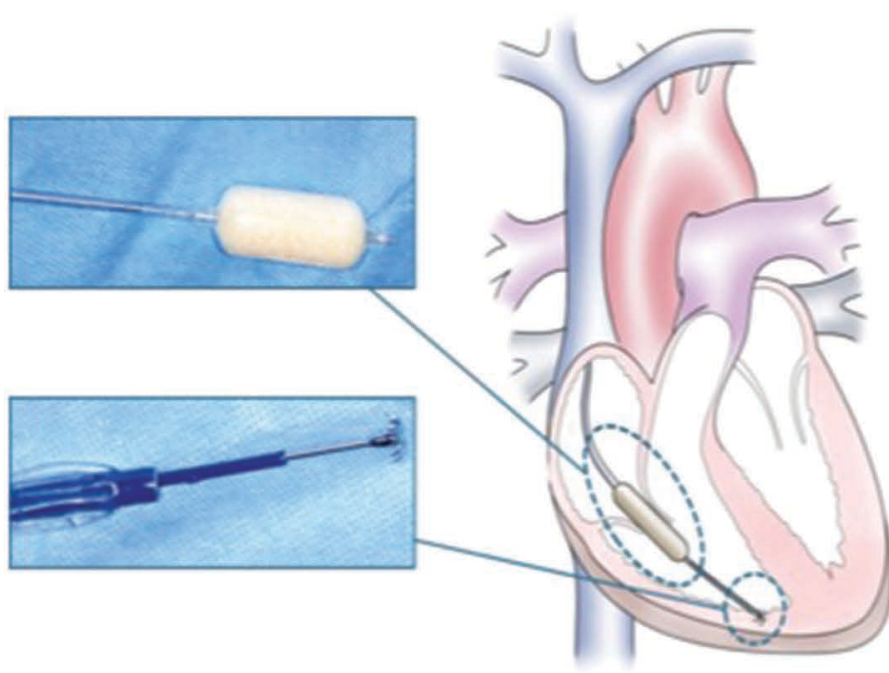

B
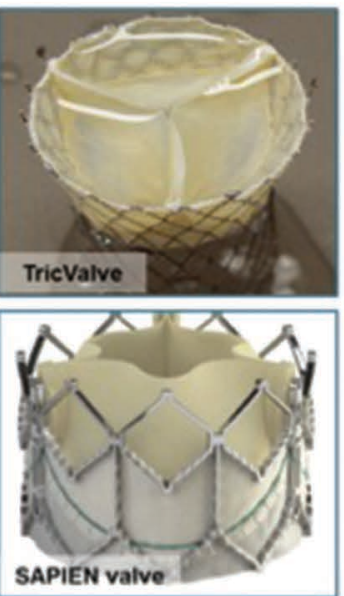

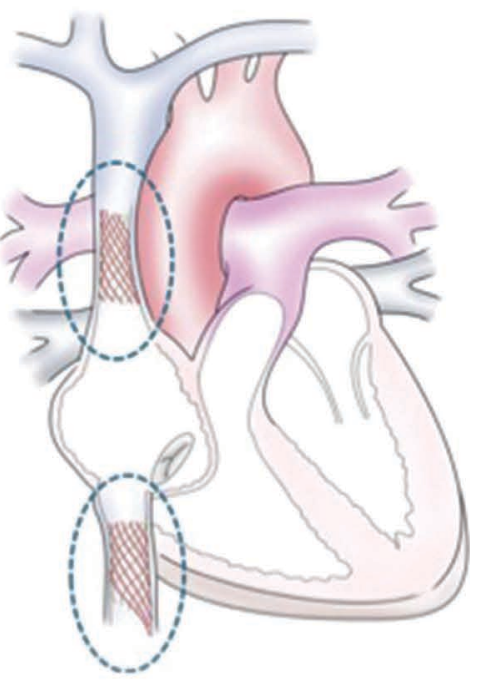

Modified from Taramasso et al. Eur Heart J. 2016 Jan 21. pii: ehv766.

2015 (unpublished data). The device was deployed temporarily during the course of valve surgery, under direct surgical vision. It was verified no interference with electrical conduction or trouble with anchoring, and the ring achieved reduction in annular diameter. A Phase ॥ study of pre-clinical procedures using transcatheter delivery began in 2016 (NCT02607527).

\section{Leaflet Repair Devices}

\section{Edge-to-edge Repair in the Tricuspid Position with the} MitraClip $®$ System

Intervention edge-to-edge repair with the MitraClip ${ }^{\circledR}$ system (Abbott vascular, Inc.) is a validated technique to treat mitral regurgitation of both degenerative and functional aetiology. The same concept has been tested in patients with severe symptomatic TR deemed to be at high risk for surgery. ${ }^{16}$ The procedure has been performed with a transjugular or transfemoral venous approach; the latter is preferred most frequently. To date, approximately 70 cases have been performed worldwide (unpublished data). Bench-test models have shown that the ideal location to place the clip to achieve the most pronounced reduction of TR in the presence of functional regurgitation is the anteroseptal commissure. ${ }^{17}$

The use of the Mitraclip system for the TV raised some specific issues due to the complexity of the TV itself, as the MitraClip system was designed specifically for the mitral valve. In the future, the technique must be refined and the device modified for dedicated use in TV disease. Combining the edge-to-edge repair with another percutaneous technique of TR annulus reduction to facilitate the clip placement may also be considered. Intraprocedural imaging remains a major issue, as TEE is commonly suboptimal. Usually a combination of TEE, transthoracic echocardiography (TTE), ICE and fluoroscopy is used to achieve suitable imaging. Fusion imaging technologies such as the EchoNavigator (Philips) have also been used to improve intraprocedural guidance.

\section{Tricuspid valve spacer}

The concept of using a valve spacer to provide a surface for native leaflet coaptation in the presence of functional regurgitation has initially been investigated in the mitral position. The FORMA Repair System (Edwards Lifesciences) consists of a spacer and a rail that is anchored at the RV apex, through a venous subclavian access (see Figure 4A). The spacer is a foam-filled polymer balloon that passively expands via holes in the spacer shaft. Two radiopaque markers help to initially position the spacer using fluoroscopy. The device is fixed at the distal end in the RV myocardium. The fixation mechanism consists of a six-pronged nitinol anchor that is designed to minimise both the risk of penetration of the epicardial surface and the prong exposure in the RV. The optimal position of the device must be perpendicular to the valve plane to allow all leaflets to coapt with the device. The device is locked proximally, and the excess rail is coiled and placed within a subcutaneous pocket. The device was applied for first-in-human experience in seven patients. ${ }^{18}$ All patients were successfully implanted with no major complication. The degree of TR was reduced by one degree in three patients and by two degrees in four patients. At 30 days, all patients but one demonstrated improvement in functional status and in quality of life. ${ }^{18}$

Further feasibility data will be obtained from a prospective registry study (Early Feasibility Study of the Edwards Tricuspid Transcatheter Repair System; NCT02471807).

\section{Caval Valve Implantation: the Caval Valve} Implantation Concept

An alternative approach to the percutaneous treatment of $T V$ is to implant a transcatheter prosthesis in the inferior vena cava (single-valve approach) or in combination with a superior vena cava valve (dualvalve approach) to prevent damage to the liver and other organs (see Figure $4 B$ ). In the presence of advanced RV dysfunction, the single-valve approach seems to be safer compared with the dual-valve approach as it is less likely to increase RV preload. The rationale for this procedure is to reduce hepatic, abdominal and peripheral venous congestion leading to amelioration of right heart failure. Different devices have been implanted using this approach and there is no consensus on what would the ideal caval valve implantation (CAVI) prosthesis be. Acute haemodynamic as well as clinical improvements at follow-up have been reported with balloon-expandable and also with self-expandable valves. 19,20 
At present, two different clinical CAVI trials are enrolling high-risk patients with severe symptomatic TR: the Treatment of Severe Secondary Tricuspid Regurgitation in Patients With Advance Heart Failure With Caval Vein Implantation of the Edwards Sapien XT Valve (TRICAVAL) study in Europe (NCT02387697) and the Heterotopic Implantation of the Edwards-Sapien XT Transcatheter Valve in the Inferior Vena Cava for the Treatment of Severe Tricuspid Regurgitation (HOVER) trial in the US (NCT02339974). The main advantage of the CAVI is that is technically easy to perform. Although preliminary results are promising, the main disadvantage of this approach is the complete absence of a clinical or surgical background. Moreover, the long-term effects of a 'ventricularisation' of the RA are unknown. Dedicated CAVI devices are under preclinical development.

\section{Conclusion}

Although only preliminary data are currently available, transcatheterbased techniques for the treatment of TR have been rapidly evolving in recent years. However, it is important to point out that at the present stage of the development of these therapies, because data are scarce, it is not possible to make any definitive conclusions around the clinical efficacy or safety. Some of these novel devices are based on well-known surgical techniques that have subsequently progressed to less-invasive approaches, others are completely new concepts that should be validated in the clinical setting. The anatomical features of the TV apparatus and right heart chambers have made transcatheter treatment of the TV challenging. Experience with aortic and mitral valve percutaneous interventions has showed us that further research is required before TV transcatheter technology can become a routine therapeutic option in routine clinical practice.

Besides technical issues, most importantly there are several clinical issues associated with these techniques. First, it is currently difficult to identify the ideal patients that could most benefit from transcatheter TV treatment, in terms of prognostic and clinical benefit.

The second important issue refers to anatomical patient selection, which relates to the TV imaging. While TTE and TEE are the most important diagnostic tool to assess TV, angio-CT is acquiring increasing importance in patient selection and procedural planning in TV percutaneous strategy. Three-dimensional echocardiography will also play an important role, as it allows accurate assessment of the morphology of the valve and of the quantification of TR.

Data from the ongoing and upcoming clinical trials should elucidate the missing information.
1. Taramasso $\mathrm{M}$, Vanermen $\mathrm{H}$, Maisano $\mathrm{F}$, et al. The growing clinical importance of secondary tricuspid regurgitation. J Am

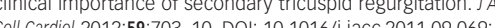
PMID: 22340261.

2. Stuge $\mathrm{O}$, Liddicoat J. Emerging opportunities for cardiac surgeons within structural heart disease. I Thorac Cardiovas surg 2006;132:1258-61. DOI: 10.1016/.j.tcvs.2006.08.049; Surg 2006;132:1258-6

3. Agricola E, Stella S, Gullace M, et al. Impact of functional tricuspid regurgitation on heart failure and death in patients tricuspid regurgitation on heart failure and death in pati
with functional mitral regurgitation and left ventricular with functional mitral regurgitation and left ventricular
dysfunction. Fur I Heart Fail 2012:14:902-8. DOl: 10.1093/eurjht/ dysfunction. Eur J Heart Fail 20

4. Joint Task Force on the Management of Valvular Heart Disease of the European Society of Cardiology (ESC); European Association for Cardio-Thoracic Surgery (EACTS), Vahanian A, Alfieri O, Andreotti F, et al. Guidelines on the management of valvular heart disease (version 2012). Eur Heart J 2012;33:2451-96. DOI: 10.1093/eurheartj/ehs109; PMID: 22922415.

5. Nishimura RA, Otto CM, Bonow RO, et al. 2014 AHA/ACC Guideline for the Management of Patients With Valvular Heart Disease: a report of the American College of Cardiology/ American Heart Association Task Force on Practice Guidelines. Circulation 2014;129:e521-643. DOI: 10.1161/ CIR.0000000000000031; PMID: 24589853

6. Kim YJ, Kwon DA, Kim HK, et al. Determinants of surgical outcome in patients with isolated tricuspid regurgitation. Circulation 2009;120:1672-8. DOI: 10.1161/ CIRCULATIONAHA.109.849448; PMID: 19822809.
7. Rodes-Cabau 」, Taramasso M, O'Gara PT. Diagnosis and treatment of tricuspid valve disease: current and future perspectives. Lancet 2016:388:2431-42. DOI: 10.1016/S01406736(16)00740-6; PMID: 27048553

8. Taramasso M, Pozzoli A, Guidotti A, et al. Percutaneous tricuspid valve therapies: the new frontier. Eur Heart J 2016: pii: ehv766. [Epub ahead of print]. DOI: 10.1093/eurheartj/ pii: ehv766. [Epub ahead
ehv766; PMID: 26802134

9. Dreyfus $G D$, Martin RP, Chan KM, et al. Functional tricuspid regurgitation: a need to revise our understanding. I Am Coll Cardiol 2015:65:2331-6. DOl: 10.1016/i.jacc.2015.04.011: PMID: 26022823.

10. Latib A, Agricola E, Pozzoli A, et al. First-in-man implantation of a tricuspid annular remodeling device for functional tricuspid regurgitation. JACC Cardiovasc Interv 2015;8:e211-4. DOI: 10.1016/j.jcin.2015.06.028; PMID: 2658562

11. Taramasso M, Latib A, Denti P, et al. Percutaneous repair of the tricuspid valve using a novel cinching device: acute and chronic experience in a preclinical large animal mode EuroIntervention 2016;12:918-25. DOI: 10.4244/EIJV1217A150; PMID: 27639745

12. Schofer J, Bijuklic K, Tiburtius C, et al. First-in-human transcatheter tricuspid valve repair in a patient with severely regurgitant tricuspid valve. I Am Coll Cardiol 2015;65:1190-5. DOI: 10.1016/j.jacc.2015.01.025; PMID: 25748096

13. Schofer J, Tiburtius C, Hammerstingl C, et al. Transfemoral tricuspid valve repair using a percutaneous mitral valve repair system. J Am Coll Cardiol 2016;67:889-90. DOI: 10.1016/ j.jacc.2015.11.047; PMID: 26892424
14. Maisano F, Taramasso M, Nickenig G, et al. Cardioband, a transcatheter surgical-like direct mitral valve annuloplasty 817-25. DOl: 10.1093/

15. Taramasso M, Guidotti A, Cesarovic N, et al. Transcatheter direct Taramasso M, Guidottl A, Cesarovic N, et al. Transcatheter direct
mitral annuloplasty with Cardioband: feasibility and efficacy trial in an acute preclinical model. Eurolintervention 2015;11:e1428-34. DOl: 10.4244/EIYY15M11 06: PMID: 26573974.

16. Hammerstingl $C$, Schueler R, Malasa M, et al. Transcatheter treatment of severe tricuspid regurgitation with the MitraCl system. Eur Heart J 2016;37:849-53. DOI: 10.1093/eurheart// ehv710; PMID: 26744457

17. Vismara R, Gelpi G, Prabhu S, et al. Transcatheter edge-toedge treatment of functional tricuspid regurgitation in an ex vivo pulsatile heart model. J Am Coll Cardiol 2016;68:1024-33. DOI: 10.1016/j.jacc.2016.06.022; PMID: 27585507.

18. Campelo-Parada F, Perlman G, Philippon F, et al. First-in-man experience of a novel transcatheter repair system for treating severe tricuspid regurgitation. J Am Coll Cardiol 2015;66. 2475-83. DOl: 10.1016/j.jacc.2015.09.068; PMID: 26653620.

19. Lauten A, Doenst T, Hamadanchi A, et al. Percutaneous bicaval valve implantation for transcatheter treatment of tricuspid regurgitation: clinical observations and 12-month follow-up. Circ Cardiovasc Interv 2014;7:268-72. DOI: 10.1161/ CIRCINTERVENTIONS.113.001033; PMID: 24737337.

20. Laule M, StangI V, Sanad W, et al. Percutaneous transfemoral management of severe secondary tricuspid regurgitation with Edwards Sapien XT bioprosthesis: first-in-man experience. J Am Coll Cardiol 2013;61:1929-31. DOI: 10.1016/ i.jacc.2013.01.070; PMID: 23500268. 